\title{
The Regulation of Prototype Foamy Virus 5 'Long Terminal Repeats and Internal Promoter by Endogenous Transcription Factors
}

\author{
Jie Wei ${ }^{\mathrm{a}}$ Yan Sun ${ }^{\mathrm{a}}$ Ting-ting Wang $^{\mathrm{a}}$ Gui Zhu ${ }^{\mathrm{a}}$ Wan-hong Liu ${ }^{\mathrm{b}}$ Xiao-hua He \\ Zhi Lia

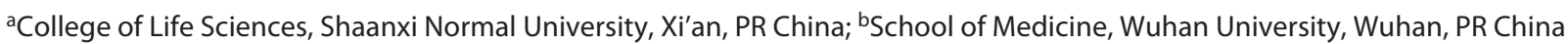

\author{
Keywords \\ Foamy virus - Tas - Viral promoters · Activator protein-1 . \\ BCL2-associated athanogene 3
}

\begin{abstract}
Background: For foamy virus, the transactivator of spumaretrovirus (Tas) could bind directly to target DNA sequences termed as Tas responsive elements and trigger the viral internal promoter (IP) and long terminal repeat (LTR) promoters. The cellular endogenous factors also play an important role in viral gene expressions. We hypothesized that except the viral transcription factor Tas, the cellular endogenous factors also affect the viral gene expression. Methods: The full length of the prototype foamy virus (PFV) genome (U21247) was used to predict the potential binding sites of the transcription factors by online software JASPAR (http://jaspar.genereg.net) and Softberry (http://linux1.softberry.com/berry. phtml?topic=index\&group=programs\&subgroup=promot er). The Dual-Luciferase ${ }^{\circledast}$ Reporter Assay System (Promega, USA) was used to confirm the relative luciferase activities of the test groups. The different representative activating
\end{abstract}

agents or inhibitors of each canonical signal pathway were used to identify the impact of these pathways on PFV 5'LTR and IP promoters. Results: The results showed different cellular endogenous factors might have respective effects on PFV 5'LTR and IP. It is worth mentioning that activator protein-1 and BCL2-associated athanogene 3, 2 kinds of vital proteins associated with NF-KB and PKC pathways, could activate the basal activity of 5'LTR and IP promoters but inhibit the Tas-regulated activity of both promoters. Furthermore, PFV Tas was identified to trigger the transcription of the NF$\mathrm{KB}$ promoter. Conclusion: NF-KB had a negative effect on $\mathrm{PFV}$ 5'LTR and IP promoter activities, the PKC pathway might upregulate $5^{\prime}$ LTR and IP promoter activities, and the JNK and NF-AT signal pathway could increase the Tas-regulated promoter activity of PFV 5'LTR. This study sheds light on the interaction between PFV and the host cell and may help utilize the viral promoters in retroviral vectors designed for gene transfer experiments.

(C) 2021 The Author(s)

Published by S. Karger AG, Basel

Yan Sun and Zhi Li contributed equally to this work as co-corresponding authors.

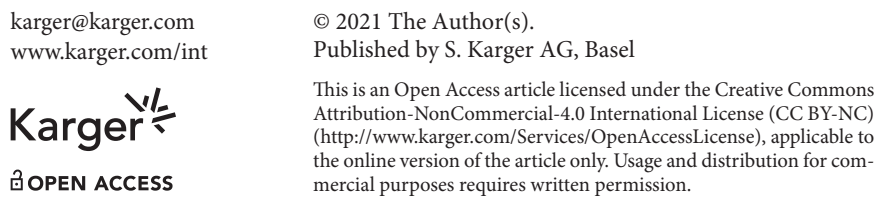

karger@karger.com www.karger.com/int

Karger $\stackrel{\text { ' }}{5}$

BOPEN ACCESS

(C) 2021 The Author(s)

Published by S. Karger AG, Basel

This is an Open Access article licensed under the Creative Commons Attribution-NonCommercial-4.0 International License (CC BY-NC) (http://www.karger.com/Services/OpenAccessLicense), applicable to the online version of the article only. Usage and distribution for commercial purposes requires written permission. 


\section{Introduction}

Foamy viruses (FVs) constitute a distinct genus of retroviruses with some special characteristics [1], such as having 1 internal promoter (IP) located in $3^{\prime}$ terminal part of env gene, apart from $5^{\prime}$ long terminal repeats (LTRs) as to other retroviruses. In the replication process of FVs, the first step is that the IP promoter initiates transcription of the transactivator of spumaretrovirus (Tas), one of the nonstructural proteins of FVs. Thus, in the early stage of FV replication, the basal activity of the IP promoter is higher than that of $5^{\prime}$ LTR promoter, which results in the accumulation of Tas inside the host cell. The second step of FV replication is that Tas protein binds to both LTR and IP promoters and then LTR triggers the expression of FV structure proteins, Gag, Pol, and Env, and also IP promotes the expression of Tas. Tas is a direct DNA-binding protein containing 2 functional domains - the N-terminal DNA-binding domain and the C-terminal activation domain - that permits the functional interaction of the transactivator with components of the cellular transcription machinery $[2,3]$. The specific DNA target sites, termed as Tas responsive elements (TREs), have been identified in 5'LTR and IP of several FVs [4]. The Tas-regulated activities of LTR and IP are much higher than their basal activities. Finally, all the FV protein expressed, and the viruses start to assembly and budding to complete the replication process.

Prototype foamy virus (PFV) is an important member of FVs. In PFV, Tas could bind directly to IP and 5'LTR to activate both of the 2 promoters, while the binding activity is cell type specific among lytic and persistent infections. Both LTR and the IP are efficiently transactivated by Tas in lytic infection, while in persistent infections, it is IP but not LTR that activated significantly by Tas [5-7]. It has been reported that PFV IP contains a minimal Tasspecific DNA target site located at $-166 /-140 \mathrm{nt}$ within the IP TRE [4]. Another study showed that the PFV $5^{\prime}$ LTR contained 3 TREs located at the positions $-360 /-342 \mathrm{nt}$, $-327 /-284 \mathrm{nt}$, and $-116 /-89 \mathrm{nt}$ [8]. While it is still unclear which one of the 3 TREs in $5^{\prime}$ LTR is the functional binding site for Tas transactivation in PFV.

Except the viral transactivator, retroviruses could also activate virus transcription by the interaction of the cellular transcription machinery. For example, NF- $\kappa \mathrm{B}$ is a master regulator of pro-inflammatory genes that involve in immunity and inflammatory response [9]. It has been verified that TNF- $\alpha$ induced HIV LTR activation in hepatocytes by activating the NF- $\mathrm{BB}$ signal pathway [10]. Recently, NF- $\kappa B$ has been found to play a role in enhanc- ing bovine foamy virus (BFV) transcription through the RelB-B Tas interaction [11]. Activator protein-1 (AP-1), another important nuclear transcription factor, could regulate the activities of a variety of cellular and viral genes [12]. The canonical AP-1 complex consists of 2 subunits, c-Jun and c-fos. The Fos-Jun heterodimer bounds to the TPA/phorbol-12-myristate-13-acetate (PMA) responsive elements with high affinity, while the individual constituents of the AP-1 complex with a lower affinity (Jun) or not at all (Fos) $[13,14]$. One AP-1 binding site in the enhancer/core element of the HIV-1 promoter was demonstrated to control the ability of HIV-1 to establish latent infection [15]. BCL2-associated athanogene 3 (BAG3), a member of the BAG co-chaperone protein family, formed a complex with the heat shock protein 70 and has been shown to localize in nuclei of cells infected by some viruses, which suggests an involvement of BAG3 in viral expression and/or replication [16]. Indeed, some reports indicated a requirement of BAG3 for virus gene expression and/or viral progeny production [17]. BAG3 could maintain an HIV-1 reservoir in infected cells that do not undergo apoptosis [18]. BAG3 has also been supposed to be activated by EGR1, a transactivator downstream ERK signal pathway $[19,20]$.

The cellular transcriptions factors mentioned above have been identified to associate with virus transcription in several retroviruses. Although PFV has 2 promoters, it has similar genome structure with other retroviruses. Thus, we hypothesized the activation of transactivator Tas on $5^{\prime} \mathrm{LTR}$ and IP promoter might definitely regulate through transcription factors, such as NF- $\mathrm{BB}, \mathrm{AP}-1$, and BAG3. In this study, the NF- $\kappa \mathrm{B}, \mathrm{AP}-1$, and BAG3 expression vectors, also a series of reporter plasmids containing different viral promoter fragments are constructed. And the relationship between PFV and host transcription factors has been analyzed according to the data based on the transfection assays.

\section{Materials and Methods}

\section{Bioinformation Analysis}

The full length of PFV genome (U21247) was used to predict the potential binding sites of the transcription factors, like Tas, AP$1, \mathrm{NF}-\kappa \mathrm{B}, \mathrm{NF}-\mathrm{AT}$, IRF1, and SRF, using the online software JASPAR (http://jaspar.genereg.net) and Softberry (http://linux1.softberry.com/berry.phtml?topic=index\&group=programs\&subgrou $\mathrm{p}=$ promoter).

\section{Construction of the Plasmids}

The pHSRV13 plasmid containing the complete molecular clone of PFV (U21247) is routinely maintained in our laboratory 
Table 1. Plasmids used in the assays

\begin{tabular}{|c|c|c|}
\hline & Primers $\left(5^{\prime}-3^{\prime}\right)$ & Enzymes \\
\hline \multicolumn{3}{|l|}{ Reporter plasmid } \\
\hline \multirow{2}{*}{ pGL3-LTR (1-1121) } & TGATACGCGTTGTGGTGGAATGCC & MluI \\
\hline & GCTGCTCGAGATTGTCATGGAATT & XhoI \\
\hline \multirow[t]{2}{*}{ pGL3-LTR (1-780) } & TGATACGCGTTGTGGTGGAATGCC & MluI \\
\hline & GACTCTCGAGCTCCCGTACAATCT & XhoI \\
\hline \multirow[t]{2}{*}{ pGL3-LTR (412-780) } & GCACACGCGTATAGAAGCTTTCAC & $M l u \mathrm{I}$ \\
\hline & GACTCTCGAGCTCCCGTACAATCT & XhoI \\
\hline \multirow[t]{2}{*}{ pGL3-LTR (440-780) } & GCACACGCGTTTTCACAATCAAC & $M l u \mathrm{I}$ \\
\hline & GACTCTCGAG $\overline{C T C C C G T A C A A T C T ~}$ & XhoI \\
\hline \multirow[t]{2}{*}{ pGL3-LTR (662-780) } & GCACACGCGTAACGCAGACAGCTT & MluI \\
\hline & GACTETCGAGCTCCCGTACAATCT & XhoI \\
\hline \multirow[t]{2}{*}{ pGL3-IP (8379-9438) } & GTCG $\overline{\text { ACGCGTATCTGTTATGGAAGG }}$ & MluI \\
\hline & GCCTTCGAGCCATGATACAATCTT & XhoI \\
\hline \multirow[t]{2}{*}{ pGL3-IP (8927-9438) } & AGCTACGCGTGTTATCTGGTTTTGG & MluI \\
\hline & GCCTCTCGAGCCATGATACAATCTT & XhoI \\
\hline \multirow{2}{*}{ pGL3-IP (8980-9438) } & CTTGACGCGTAGCATCGTGACTGTT & MluI \\
\hline & GCCTCTCGAGCCATGATACAATCTT & XhoI \\
\hline \multirow[t]{2}{*}{ pGL3-IP (9019-9438) } & CTTCACGCGTTGCTTTGGACTGGAC & MluI \\
\hline & GCCTETCGAGCCATGATACAATCTT & XhoI \\
\hline \multirow[t]{2}{*}{ pGL3-IP (8379-9195) } & GTCGACGCGTATCTGTTATGGAAGG & $M l u \mathrm{I}$ \\
\hline & GTTCCTCGAGTCAGCTTTTGCTCTT & XhoI \\
\hline \multirow[t]{2}{*}{ pGL3-IP (8927-9195) } & AGCTACGCGTGTTATCTGGTTTTGG & MluI \\
\hline & GTTCCTCGAGTCAGCTTTTGCTCTT & XhoI \\
\hline \multirow[t]{2}{*}{ pGL3-IP (8980-9195) } & CTTGACGCGTAGCATCGTGACTGTT & MluI \\
\hline & GTTCTCGAGTCAGCTTTTGCTCTT & XhoI \\
\hline \multirow[t]{2}{*}{ pGL3-IP (9019-9195) } & CTTCACGCGTTGCTTTGGACTGGAC & MluI \\
\hline & GTTGCTCGAGTCAGCTTTTGCTCTT & XhoI \\
\hline \multicolumn{3}{|l|}{ Expression plasmids } \\
\hline \multirow[t]{2}{*}{ pCI-Tas } & CGACACGCGTTATCATGGATTCCTAC & MluI \\
\hline & CATA $\overline{\text { GTCGACCTGACAGCAAGTTAG }}$ & SalI \\
\hline \multirow[t]{2}{*}{ pcDNA-fos } & AGCGAAGCTTATGATGTTCTCGGGC & HindIII \\
\hline & ATCGGAATTCTCACAGGGCCAGCAG & EcoRI \\
\hline \multirow[t]{2}{*}{ pcDNA-jun } & GTCGAAGCTTATGACTGCAAAGAT & HindIII \\
\hline & CGGCGAATTCTCAAAATGTTTGC & EcoRI \\
\hline \multirow[t]{2}{*}{ pcDNA-BAG3 } & TCTCAAGCTTAGCATGAGCGCCGCC & HindIII \\
\hline & TGCA $\overline{\text { GAATTCCTACGGTGCTGCTGG }}$ & EcoRI \\
\hline
\end{tabular}

Tas, transactivator of spumaretrovirus; BAG3, BCL2-associated athanogene 3; IP, internal promoter; LTR, long terminal repeat.

(kindly offered by Prof. Wenxin Li in Wuhan University). Different fragments of $5^{\prime}$ LTR and IP were amplified using pHSRV13 as the template using the primers summarized in Table 1 . Then, the amplified products were digested with the specific enzymes and subcloned into the promoter-less luciferase reporter vector pGL3basic (Promega, USA) to construct series of LTR-Luc and IP-Luc plasmids (Fig. 1).

The open reading frame of Tas protein was amplified using pHSRV13 as the template too. Then, the fragment was subcloned into pCI-neo vector (Promega, USA) to construct the expression vector pCI-Tas. The templates for amplifying the open reading frames of Fos, Jun, and BAG3 were the cDNA clones maintained in our laboratory containing the respective gene sequences (NM_005252, NM_002228.3, and NM_004281). The expression vectors pcDNA-fos, pcDNA-jun, and pcDNA-BAG3 were constructed based on the expression vector pcDNA3.1(+) (Invitrogen, USA) using the same strategy.

\section{Western Blot Analysis}

293 cells are routinely maintained in Dulbecco's modified Eagle's medium supplemented with $10 \%$ fetal bovine serum, $100 \mathrm{IU} /$ $\mathrm{mL}$ penicillin, and $100 \mathrm{mg} / \mathrm{mL}$ streptomycin at $37^{\circ} \mathrm{C}$ in a humidified atmosphere with $5 \% \mathrm{CO}_{2}$. The plasmid pcDNA-BAG3 (75 or $150 \mathrm{ng}$ ) was transfected into the cells using lipofectamine 2000 (Invitrogen, USA) according to manufacturer's instructions. The plasmids pcDNA-fos (50 or $100 \mathrm{ng}$ ) and pcDNA-jun (50 or 100 ng) were co-transfected, as described for pcDNA-BAG3 previously. At 48-h post transfection, cells were harvested and lysed in pro- 

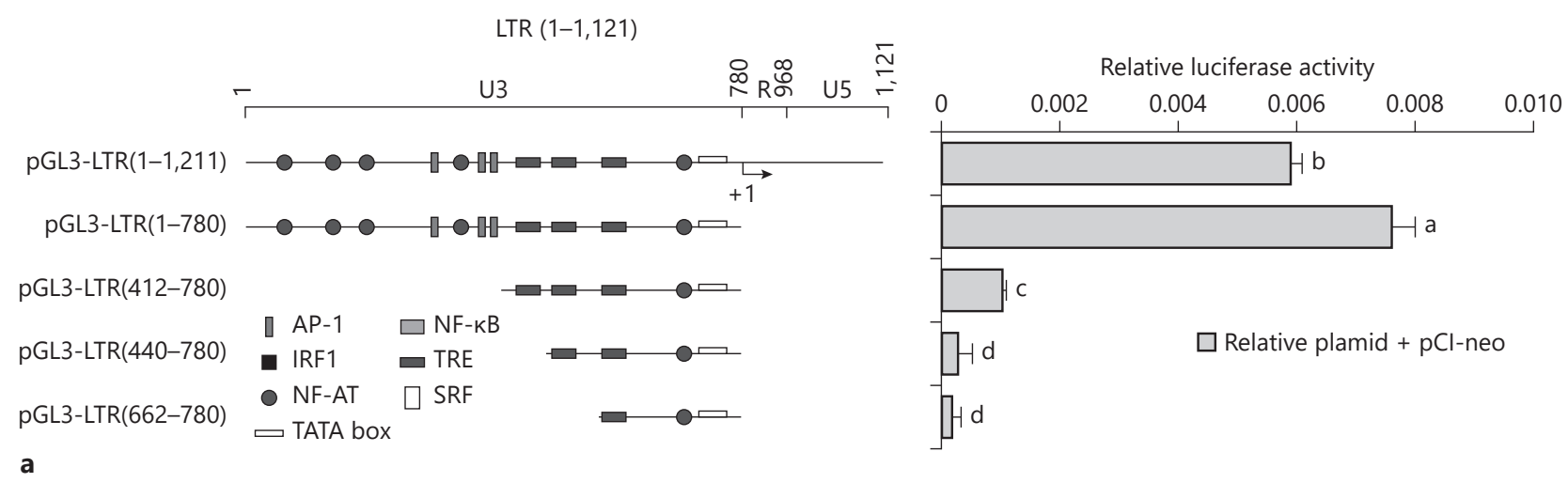

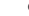

pGL3-IP (8,379-9,438)

pGL3-IP $(8,927-9,438)$

pGL3-IP $(8,980-9,438)$

pGL3-IP (9,019-9,438)

pGL3-IP $(8,379-9,195)$

pGL3-IP (8,927-9,195)

pGL3-IP $(8,980-9,195)$

pGL3-IP $(9,019-9,195)$

b

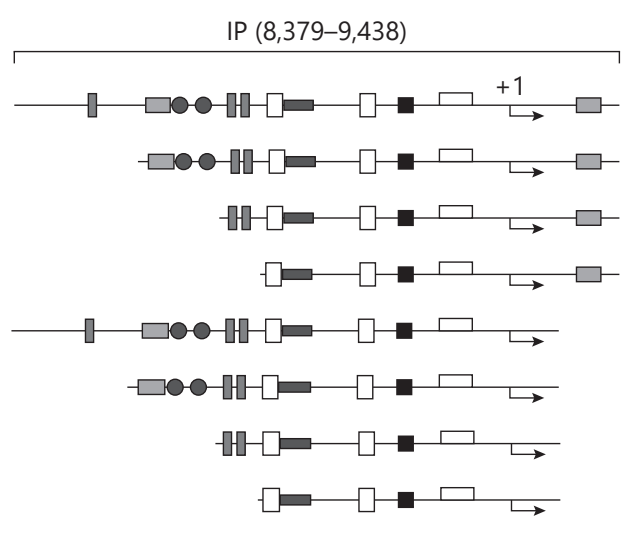

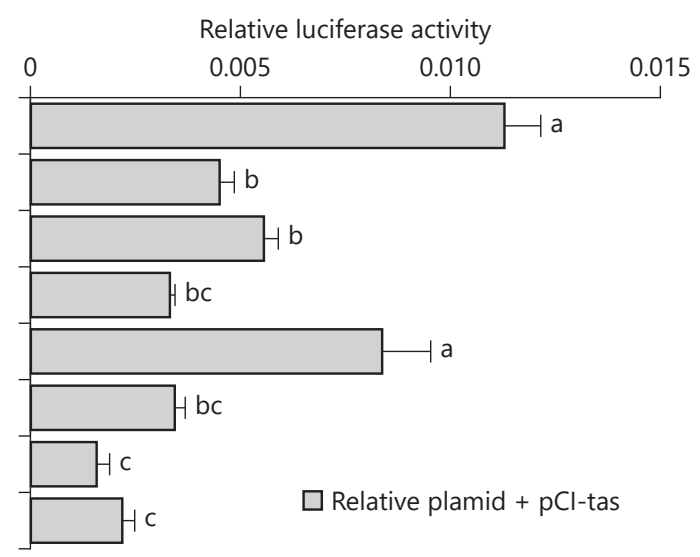

Fig. 1. Schematic diagrams of different PFV 5'LTR (a) and IP (b) fragments used to construct the luciferase reporter vectors were shown on the left part of the figure. The " +1 " indicates the transcription start site. The predicted transcription factors are labeled by different shapes which are annotated in the figure. The basal promoter activities of different PFV 5'LTR constructs (a) and IP constructs (b) were shown on the right part of the figure. The let-

tein lysing buffer (50 mM Tris- $\mathrm{HCl}$ [pH 7.4], $150 \mathrm{mM} \mathrm{NaCl}, 1 \%$ NP-40, 0.1\% SDS). After being boiled for $10 \mathrm{~min}$ and centrifuged, the supernatant was loaded to SDS-PAGE gel. Equal quantities of total proteins were loaded per lane. Then the proteins were transferred to PVDF membrane and incubated with anti-BAG3 (Bioss, China) and anti-fos (Bioss) polyclonal antibodies, respectively. The horseradish peroxidase-conjugated goat anti-rabbit immunoglobulin G (Bioss) was used as secondary antibody. Blots were developed using the Immuno-star horseradish peroxidase substrate (Genshare Biological, China) according to the manufacturer's protocol.

\section{Cell Culture and Transfection}

Three cell lines, U87-MG (human malignant glioma cells), Hela (cervix adenocarcinoma epithelial cells), and HT1080 (human fibrosarcoma cells) were maintained in Dulbecco's modified Eagle's medium (12100; Solarbio, China) supplemented with $10 \%$ ters labeled on the top of columns indicate the significant difference. For example, the same letters labeled on 2 columns mean there is no significant difference between the 2 promoter fragments, while different letters mean there is significant difference between the 2 promoter fragments. PFV, prototype foamy virus; LTR, long terminal repeat; IP, internal promoter; TRE; Tas responsive elements; AP-1, activator promoter-1.

fetal bovine serum (CP17-1616; Capricorn, Germany) and 1\% penicillin, and streptomycin in $5 \% \mathrm{CO}_{2}$ at $37^{\circ} \mathrm{C}$. The transient transfections were carried out using the 3 cell lines, respectively, to examine whether the results were consistent in different cells.

For transfection, $1-2 \times 10^{4}$ cells were seeded in each well of the 96-well plates. To detect the LTR and IP activities, 33 ng of reporter plasmids plus $163 \mathrm{ng}$ of pCI-Tas, or pCI-neo, were co-transfected using lipofectamine 2000 (Invitrogen) according to manufacturer's instructions. At the same time, 4 ng of pRL-SV40, expressing Renilla luciferase, was used as the internal control to ensure transfection efficiency. All the transfections were done in triplicate.

To find out whether Tas could affect the pathways, several reagents were used in the transfection experiments (Table 2). The amounts of the activators or the inhibitors used in the tests were repeated after published reports to achieve specific activation or inhibition of each pathway (summarized in Table 2). None of the 
Table 2. Activators or inhibitors used in the transient transfection experiments $[1,12,13,19,23,33]$

\begin{tabular}{llll}
\hline Drug & Target & Function & Reference \\
\hline PMA & PKC signal pathway & Activator & {$[1]$} \\
STS & PKC signal pathway & Inhibitor & {$[33]$} \\
TNF- $\alpha$ & NF- $\kappa$ B signal pathway & Activator & {$[23]$} \\
PDTC & NF- $\kappa$ B signal pathway & Inhibitor & {$[13]$} \\
SP600125 & JNK signal pathway & Inhibitor & {$[19]$} \\
CsA & NF-AT signal pathway & Inhibitor & {$[12]$} \\
\hline
\end{tabular}

PMA, phorbol-12-myristate-13-acetate.

inhibitors or activators, at the indicated concentrations, affected the viability of the cells. All the transfections were done in triplicate.

To determine the functions of Fos-Jun heterodimer and BAG3, which involve in $\mathrm{PKC}$ and NF- $\mathrm{KB}$ pathways, respectively, co-transfection has been carried out following the exact same procedures as described in previous co-transfection experiments performed in our research. The amounts of the plasmids used were labeled in the figures. All the transfections were done in triplicate.

To reveal the functions of Tas in regulating the expression of NF- $\kappa$ B, pNFкB-TA-luc plasmid (Beyotime Biotechnology Co., China) was purchased in which the expression level of $L u c$ gene may reflect the transcription activity of NF- $\kappa B$. For transfection, HT1080 cells were prepared as described previously. Then $33 \mathrm{ng}$ of pNFkB-TA-luc plus 163 ng of pCI-Tas, or pCI-neo, were cotransfected. At the same time, $4 \mathrm{ng}$ of pRL-SV40 was used as internal control to ensure transfection efficiency. All the transfections were done in triplicate.

\section{Dual-Luciferase Reporter Assays}

At 48-h after transfection, cells were washed with PBS and then lysed with passive lysis buffer supplied in the Dual-Luciferase ${ }^{\circledR}$ Reporter Assay System (Promega, USA). Then the firefly and Renilla luciferase activities were determined using the kit according to the manufacturer's instructions. The firefly luciferase activity of each sample was normalized by Renilla activity. The relative luciferase activities of the test groups were calculated as the assumption that the luciferase activity of the control group was 1.0.

\section{Statistical Analysis}

Data were presented as the mean $\pm \mathrm{SD}(n=3)$. All experiments were performed 3 times independently. A 2-sample $t$ test was used to evaluate statistical significance $(p<0.05)$.

\section{Results}

\section{The Basal Promoter Activities of PFV 5'LTR and IP}

The different LTR-luc and IP-luc plasmids were transfected into cell lines to test the basal promoter activities of different fragments. The results indicated that the basal promoter activities of different PFV 5'LTR fragments were lower than that of different PFV IP fragments (Fig. 1). These results confirmed the previous reports that in the early stage of virus replication, PFV IP initiated the expression of Tas which further activated PFV 5'LTR and IP, respectively.

On the other hand, the diversity promoter activities of different PFV 5'LTR fragments and PFV IP fragments indicated that the endogenous transcription factors might be involved in the PFV 5'LTR and PFV IP basal promoter activities. Further computer predictions indicate that the AP-1 binding sites, the NF-AT binding sites, and many other transcription factors binding sites exist in the PFV 5'LTR and IP.

As the NF- $\kappa$ B pathway was a main pathway which was supposed to be involved in the virus replication, the reporter plasmids containing the LTR fragment $1-1121$ and 662-780 were chosen to figure out whether the NF- $\kappa B$ pathway would affect the basal promoter activity of PFV $5^{\prime}$ LTR (Fig. 2a). But the addition of TNF- $\alpha$, the NF- $\kappa B$ pathway activator, did not affect the activities of LTR (11121 ) and LTR (662-780) which indicated that activation of the NF- $\mathrm{KB}$ signal pathway did not pose any effect on the basal activity of $5^{\prime} \mathrm{LTR}$ promoter. But the addition of PDTC, a specific NF- $\kappa$ B pathway inhibitor, resulted in the upregulation of LTR (1-1121) promoter activity, which suggested that the NF- $\kappa B$ signal pathway performed negative effect on the basal PFV 5'LTR promoter activity. According to the prediction, $3 \mathrm{AP}-1$ binding sites were found in the LTR fragment 1-412. Therefore, LTR (1-780) and LTR (412-780) reporter plasmids were used to test whether PKC and JNK signal pathways could affect PFV 5'LTR basal promoter activity. The addition of PMA, which could activate several isoforms of $\mathrm{PKC}$, resulted in the upregulation of LTR (1-780) activity. What's more, PMA did not show any significant effect on LTR (412-780) promoter activity, which might be due to no AP-1 binding sites on the LTR fragment (412-780). When STS, a specific inhibitor of the PKC signal pathway was introduced into the tests, LTR (1-780) basal activity was decreased significantly. The SP600125 and CaA were JNK signal pathway inhibitor and NF-AT pathway inhibitor, respectively. The basal activities of LTR (1-780) and LTR (412-780) changed little under the treatment of SP600125, also the basal activity of LTR (662-780) changed little when treated by CaA, which indicated that JNK and NF-AT signal pathways did not involve in the basal promoter activity of PFV $5^{\prime} \mathrm{LTR}$.

For PFV IP, 1 NF- $\mathrm{kB}$ binding site was found between 8927 and 8980 nt on IP promoter. IP (8379-9195) and IP (8980-9195) luciferase reporters were used to figure out whether the NF- $\mathrm{kB}$ signal pathway had effect on IP promoter activity (Fig. 2b). The pathway activator TNF- $\alpha$ did 


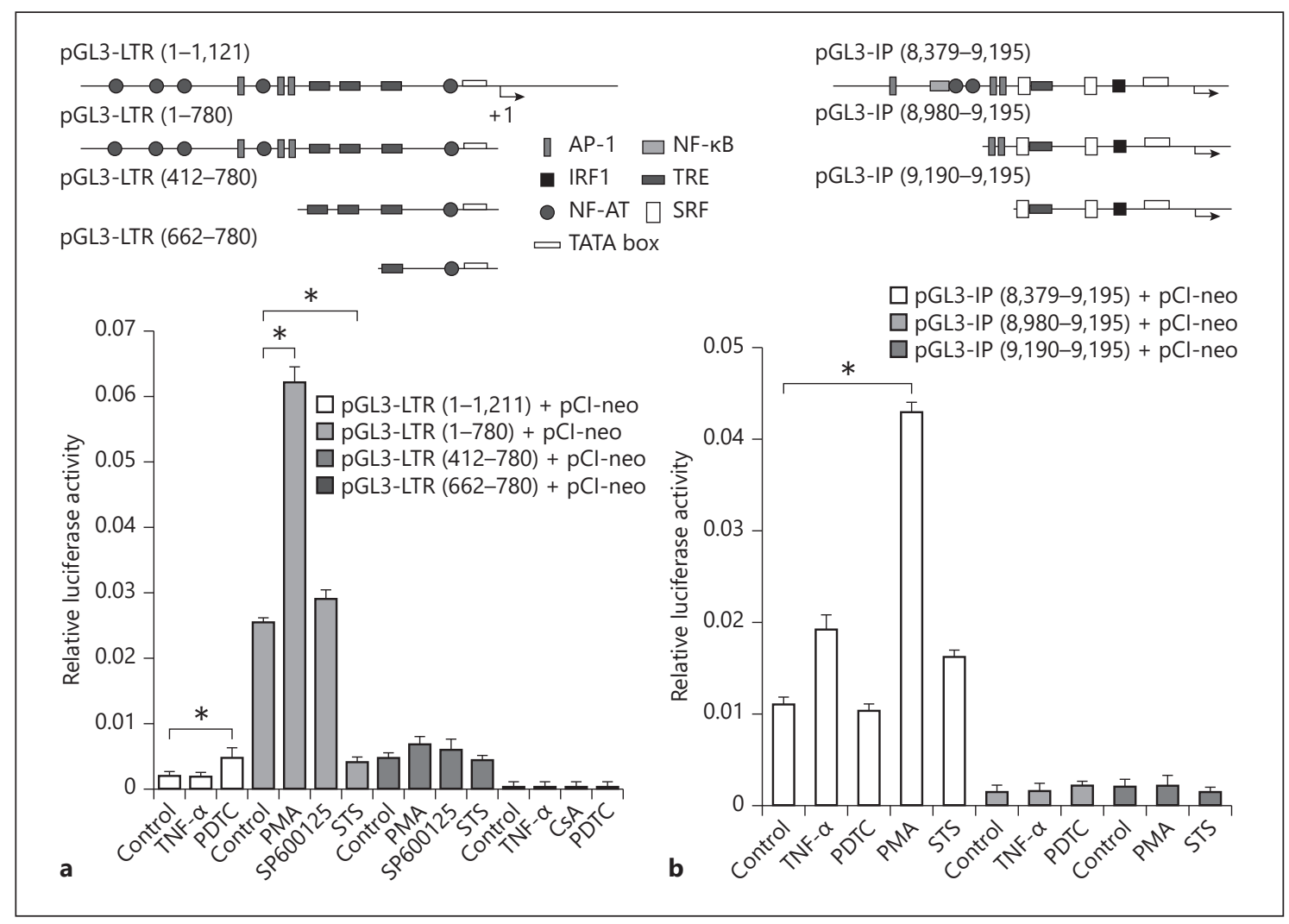

Fig. 2. Basal promoter activities of PFV $5^{\prime}$ LTR promoter vectors (a) and IP promoter vectors (b) treated with different reagents which may activate or inhibit specific cellular signal pathways. TNF- $\alpha$, NF- $\kappa$ B pathway activator; PDTC, NF- $\kappa B$ pathway inhibitor; PMA, PKC pathway activator; STS, PKC pathway inhibitor;
SP600125, JNK pathway inhibitor; CsA, NF-AT pathway inhibitor; PFV, prototype foamy virus; LTR, long terminal repeat; IP, internal promoter; TRE; Tas responsive elements; AP-1, activator promoter-1; PMA, phorbol-12-myristate-13-acetate. Asterisks indicate significant difference. not affect the basal IP promoter activity. Also, the NF- $\kappa \mathrm{B}$ inhibitor PDTC did not affect the basal IP promoter activity. Three AP-1 binding sites were predicted between 8379 and $9091 \mathrm{nt}$ on IP promoter. Thus, IP (8379-9195) and IP (9091-9195) luciferase reporters were used to examine whether PKC and JNK pathways were associated with PFV IP basal activity. When PMA was added, IP (8379$9195)$ promoter activity was significantly increased. However, the STS did not affect the PFV IP basal activity. Also, the JNK pathway inhibitor SP600125 did not affect the IP (8379-9195) and IP (9091-9195) promoter activities (data not shown). This indicated that PKC and JNK pathways did not involve in PFV IP basal activity.

\section{The Activated Promoter Activities of PFV 5'LTR and}

IP by Tas

In order to deeply explore what the effects of cell endogenous transcription factors performed during the activation of $5^{\prime} \mathrm{LTR}$ and IP by Tas, co-expression of pCI-Tas and series of promoter fragments were carried out to examine the Tas-activated PFV 5'LTR and IP promoter activities. It was obvious that in every tested group, the PFV $5^{\prime}$ LTR activities triggered by Tas were significantly much higher than the basal activities (Fig. 3a). Deletion of RU-5 region resulted in remarkable increase of the PFV 5 LTR activity, which indicated that RU-5 region might exert negative effect on Tas-regulated PFV $5^{\prime}$ LTR activity. When the 440-662 fragment of PFV 5'LTR was deleted, Tas-regulated activity of LTR (662-780) decreased dramatically. This suggested that the second TRE might pose very important function in upregulating the activity of PFV 5'LTR. The Tas-regulated PFV IP activities were measured in the same way (Fig. 3b). Similar to $5^{\prime}$ LTR, the Tas-activated PFV IP activities were significantly much higher than its basal activities in each group. What's more, deletion of the fragment 9195-9438 demonstrated great reduction of Tas-regulated IP activities. According to the prediction, 1 canonical NF- $\kappa \mathrm{B}$ binding site was 

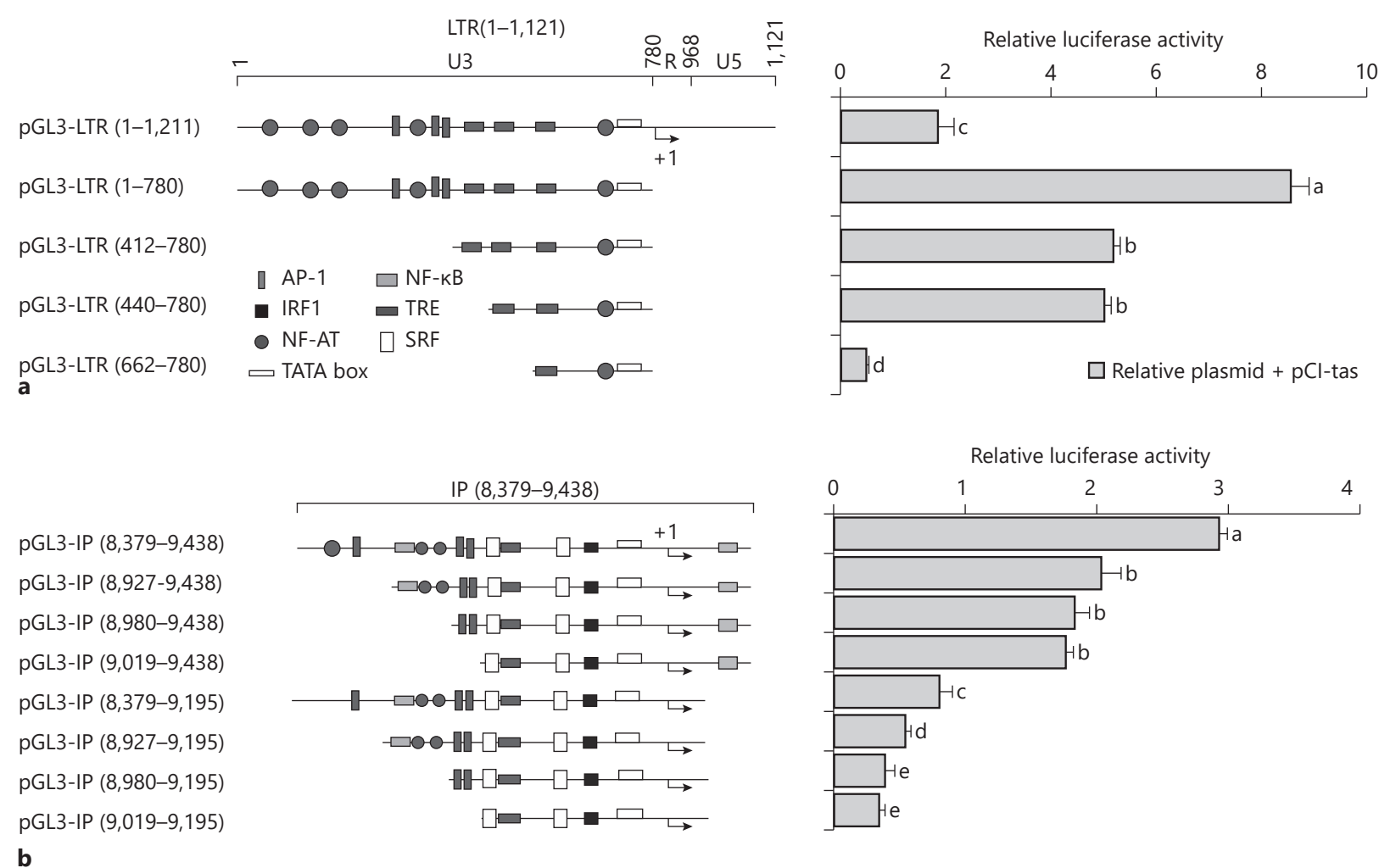

Fig. 3. Schematic diagrams of different PFV 5'LTR (a) and IP (b) fragments used to construct the luciferase reporter vectors were shown on the left part of the figure. The " +1 " indicates the transcription start site. The predicted transcription factors are labeled by different shapes which are annotated in the figure. The activated promoter activities of different PFV $5^{\prime}$ LTR constructs (a)

found on this part of IP promoter. Thus, this binding site might play an important role in IP promoter activity. At the same time, the differential promoter activities between the different IP fragments indicated many endogenous transcription factors might take part in the Tasregulated IP activity.

As the NF- $\kappa$ B pathway was a main pathway which was involved in the virus replication, NF- $\kappa \mathrm{B}$ pathway activator TNF- $\alpha$ and NF- $\kappa \mathrm{B}$ pathway inhibitor PDTC were used to figure out whether the NF- $\kappa B$ pathway would affect Tas-regulated 5'LTR activity (Fig. 4a). The addition of TNF- $\alpha$ did not affect the activities of LTR (1-1121) and LTR (662-780), while the existing of PDTC resulted in the upregulation of LTR (1-1121) and LTR (662-780) promoter activity. This suggested that the NF- $\kappa B$ signal pathway performed negative effect on Tas-regulated PFV $5^{\prime}$ LTR promoter activities. According to the prediction, 3 and IP constructs (b) were shown on the right part of the figure. The letters labeled on the top of columns indicate the significant difference. PFV, prototype foamy virus; LTR, long terminal repeat; IP, internal promoter; TRE, Tas responsive elements; AP-1, activator promoter-1; PMA, phorbol-12-myristate-13-acetate.

AP-1 binding sites were found in the LTR fragment 1-412. Therefore, LTR (1-780) and LTR (412-780) reporter plasmids were used to test whether PKC and JNK signal pathway could affect Tas-regulated PFV 5'LTR activity. The existing of PMA, which could activate several isoforms of PKC, resulted in the upregulation of LTR (1780) activity. This revealed that PKC pathway might activate PFV 5'LTR. What is more, PMA did not show any significant effect on LTR (412-780) promoter activity in the presence of Tas protein, which confirmed the prediction that AP-1 binding sites were missed in LTR (412$780)$ plasmid. While the activities of LTR (1-780) and LTR (412-780) had no changes under the treatment of STS, a specific inhibitor of PKC signal pathway. The SP600125 was a specific JNK signal pathway inhibitor. The activities of LTR (1-780) decreased dramatically under the treatment of SP600125. These results indicated 


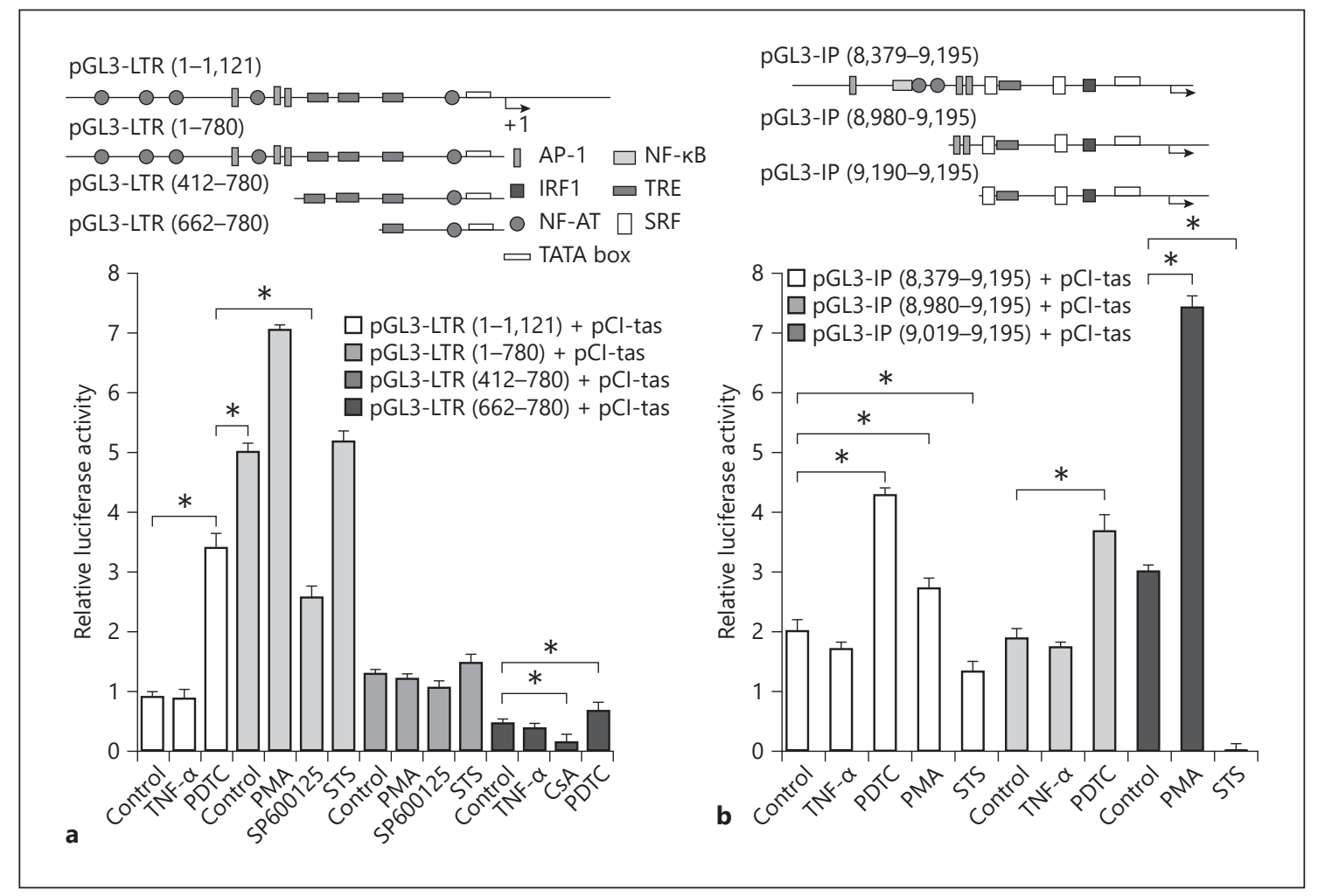

Fig. 4. Tas-regulated promoter activities of PFV 5'LTR promoter vectors (a) and IP promoter vectors (b) treated with different reagents which may activate or inhibit specific cellular signal pathways. TNF- $\alpha$, NF- $\kappa$ B pathway activator; PDTC, NF- $\kappa$ B pathway inhibitor; PMA, PKC pathway activator; STS, PKC pathway in- hibitor; SP600125, JNK pathway inhibitor; CsA, NF-AT pathway inhibitor; PFV, prototype foamy virus; LTR, long terminal repeat; IP, internal promoter; TRE, Tas responsive elements; AP-1, activator promoter-1; PMA, phorbol-12-myristate-13-acetate. Asterisks indicate significant difference. that JNK signal pathway might involve in the Tas-regulated promoter activity of PFV 5'LTR. CsA, inhibitor of NF-AT pathway, significantly inhibited Tas-activated LTR (662-780) activity in our study. Further analysis showed that 1 perfect NF-AT binding site was found right upstream of TATA box of PFV LTR. This provoked the thought that Tas could trigger the activation of $5^{\prime} \mathrm{LTR}$ through transcription factor NF-AT.

For PFV IP, 1 NF- $\kappa B$ binding site was found between 8927 and $8980 \mathrm{nt}$ on IP promoter. IP (8379-9195) and IP (8980-9195) luciferase reporters were used to figure out whether the NF- $\mathrm{kB}$ signal pathway had effect on IP promoter activity (Fig. 4b). The results indicated that the pathway activator TNF- $\alpha$ did not affect the IP promoter activities. While when NF- $\kappa B$ inhibitor PDTC was used, the activities of IP (8379-9195) and IP (8980-9195) increased dramatically than the control groups. So, we speculated the NF- $\kappa B$ signal pathway performed negative effect on Tasregulated IP promoter activity. Also, 3 AP-1 binding sites were predicted between 8379 and 9019 nt on IP promoter.
When PMA was added, IP (8379-9195) and IP (90919195) promoter activities were significantly increased and when STS added, the promoter activities decreased dramatically. Thus, the PKC pathway was identified to affect the activated PFV IP activity. The JNK pathway inhibitor SP600125 did not affect the IP (8379-9195) and IP (90919195) promoter activities with Tas protein presenting (data not shown). The JNK signal pathway seemed to have no effect on the Tas-regulated PFV IP activity.

\section{AP-1 and BAG3 Were Essential for the Regulation of} PFV 5'LTR and IP Promoter

The canonical AP-1 complex consisted of 2 subunits, c-Jun and c-Fos. With specific BAG3 and c-Fos antibodies to detect the expression of BAG3 and AP-1, it had been revealed that the constructed expression plasmids $\mathrm{pcD}$ NA-BAG3, pcDNA-fos, and pcDNA-jun could successfully express in mammalian cells (online suppl. material 1; see www.karger.com/doi/10.1159/000517539 for all online suppl. material). The previous results suggested 
Fig. 5. Promoter activities of PFV 5'LTR and IP regulated by AP-1 (a) and BAG3 (b). The plasmids pcDNA-jun and $\mathrm{pcD}$ NA-fos were used to express the 2 subunits of the heterodimer AP-1. The plasmid pcDNA-BAG3 was used to express the BAG3 protein. At the bottom of each column, the amount (nanogram) of the plasmids co-transfected in each group was labeled. PFV, prototype foamy virus; LTR, long terminal repeat; IP, internal promoter; TRE, Tas responsive elements; AP-1, activator promoter-1; PMA, phorbol-12-myristate-13-acetate; BAG3, BCL2-associated athanogene 3. Asterisks indicate significant difference.
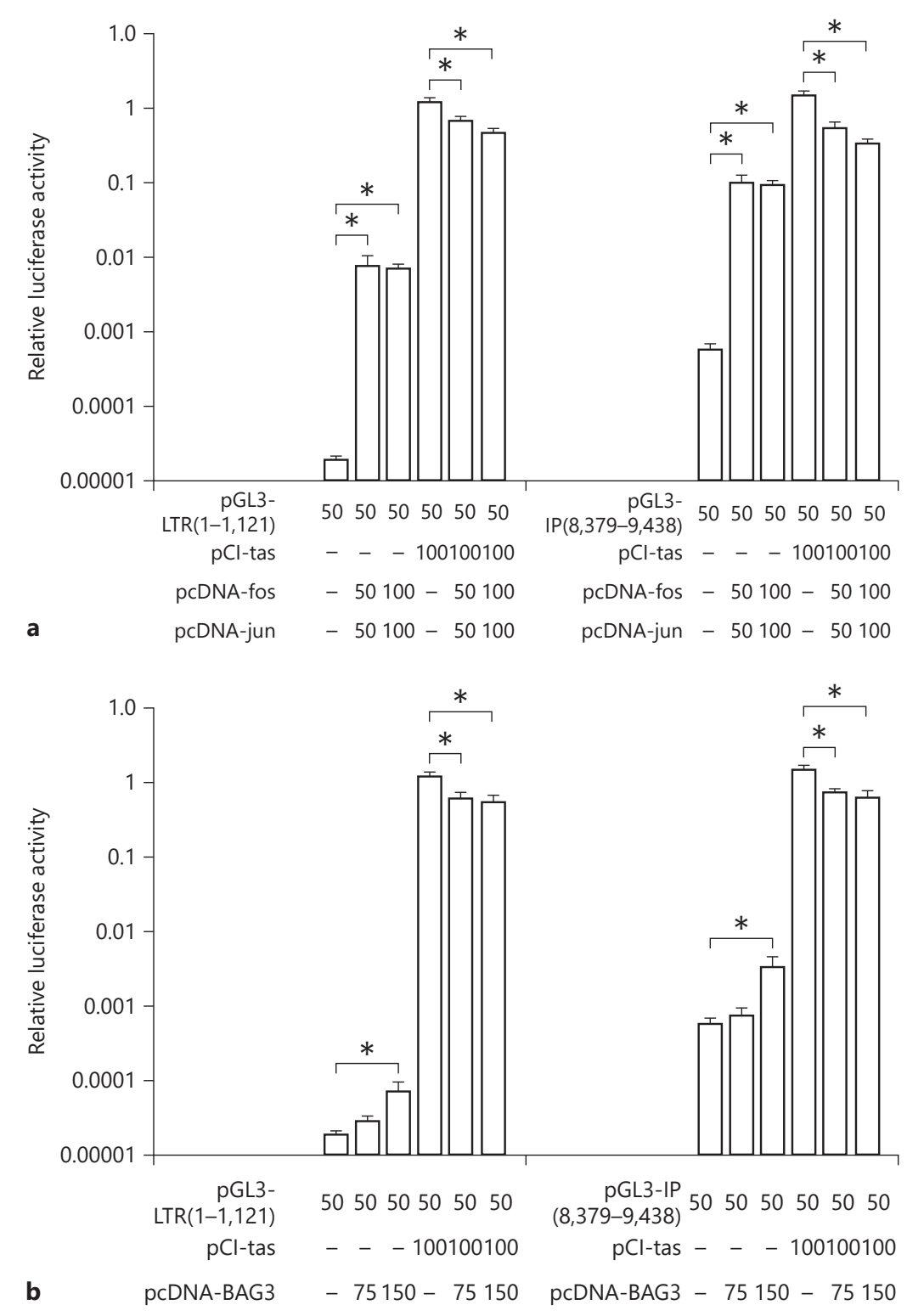

that PKC signal pathway involved in PFV 5'LTR and IP promoter activity. While whether AP-1, an important transcription factor belonging to the $\mathrm{PKC}$ pathway, could pose impact on the activities of PFV $5^{\prime}$ LTR and IP were still unclear. Our data showed that the AP-1 (the Fos-jun heterodimer) significantly increased the basal activity of PFV 5'LTR promoter but decreased the Tas-regulated 5'LTR activity (Fig. 5a). The similar results had been obtained in the experiments with AP-1 and PFV IP promoter that AP-1 upregulated the basal activity of PFV IP but downregulated the Tas-activated IP activity (Fig. 5a).
Moreover, the effect of AP-1 on LTR and IP promoter did not act in a dose-dependent way.

BAG3 was an important molecule acted upstream of NF- $\kappa \mathrm{B}$. The results revealed that BAG3 protein increased the basal activity of PFV 5'LTR promoter when Tas was absent. However, the Tas-regulated activity of $5^{\prime} \mathrm{LTR}$ was reduced by BAG3. Similar with PFV 5'LTR, BAG3 could activate the basal activity of IP promoter but inhibit the Tas-activated IP activity. Thus, it was suggested that BAG3 exhibited the similar regulation activity to AP-1 (Fig. 5b). 


\section{Discussion}

The LTR of FVs contains U3, R, and U5 region. In our experiment, deletion of R-U5 region of PFV 5'LTR resulted in upregulation of $5^{\prime}$ LTR promoter activity, which was consistent with the previous studies about SFV-1 and SFV-3 R-U5 region [21, 22]. Thus, it is a general characteristic in FVs that R-U5 region could downregulate the $5^{\prime}$ LTR promoter activity. The $5^{\prime} \mathrm{LTR}$ also contained the important TRE that were the target sites for Tas protein [23]. Usually, there are 2 or 3 TREs in FVs which are vital either for starting of transcription or acting as a typical inducible enhancer during Tas activation, while it is surprisingly to find that the DNA sequences sufficient for Tas DNA binding in vitro were insufficient in vivo [5]. This confirmed our hypothesis that other cellular DNAbinding proteins may play an important role during the activation of promoters by Tas. Then, the transcription factors were predicted by online analysis software and 5 NF-AT binding sites, 3 AP-1 binding sites were predicted in the LTR sequence while 2 NF-AT binding sites, 3 AP- 1 binding sites, 1 IRF1 binding site, and 2 NF- $\kappa B$ binding sites were also predicted in the IP sequence (online suppl. Material 2). It was reported that NF- $\kappa B$ could be inhibiting Tas transactivation of PFV LTR and IP [24], but the further molecular mechanism was still unknown. According to our results, only the second TRE in PFV $5^{\prime} \mathrm{LTR}$ $\mathrm{U} 3$ region was the functional target site for Tas. As the sequences of FV LTRs are not consistent, and the number of TREs may vary among different FVs, it could be hypothesized the phenomenon that only 1 functional TRE in PFV was unique.

The full length of PFV genome (U21247) was used to predict the potential binding sites of the transcription factors, like Tas, AP-1, NF- $\kappa B$, NF-AT, IRF1, and SRF, using online software JASPAR (http://jaspar.genereg.net) and Softberry (http://linux1.softberry.com/berry.phtml?topi $\mathrm{c}=$ index\&group $=$ programs\&subgroup $=$ promoter).

NF- $\kappa B$ binding sites were not found in PFV 5 'LTR sequence when performing analysis in silico. Consistent with the bioinformatics analysis, the activation of the NF$\kappa \mathrm{B}$ signal pathway by TNF- $\alpha$ did not alter the PFV $5^{\prime} \mathrm{LTR}$ transcriptional activity with or without Tas protein. However, when NF- $\kappa$ B signal pathway inhibitor PDTC was used, PFV 5'LTR activity was significantly increased compared to the group without PDTC. These results suggested that the NF- $\kappa \mathrm{B}$ signal pathway might exert negative effect on PFV 5'LTR indirectly. Further studies indicated that PFV Tas could trigger the transcription of NF$\kappa \mathrm{B}$ promoter (online suppl. Material 3). Thus, we drew a schematic to explain the relationship between PFV Tas and NF- $\kappa \mathrm{B}$ signal pathway: at the initiation of PFV infection, the expression level of PFV Tas protein was maintained at a low level, and the cells-maintained the NF- $\mathrm{KB}$ pathway at the ordinary level. And then the accumulation of Tas protein upregulated the PFV LTR, and at the same time, upregulated the NF- $\kappa B$ pathway which further results in the inhibition of the PFV LTR promoter. The combination of the 2 opposite effects resulted in the low level of viral proliferation and then the further consequence is the adaption for PFV to the immune approaches of the host. What is more, it may help us to complain the paradox that there is no pathogenicity of PFV in vivo but significant cytolethal effect in vitro. The similar phenomenon had been identified when studying the relationship between the PFV IP promoter and NF- $\kappa$ B pathway, as the inhibition of the NF- $\mathrm{BB}$ pathway was verified to increase the Tas-induced activity of the IP promoter. Thus, we might draw a conclusion that the NF- $\kappa B$ signal pathway played a negative role in PFV replication. However, even though the functional NF- $\kappa \mathrm{B}$ binding site was not been found on BFV LTR, it was identified that the cellular RelB may interact with BFV Tas, which enhanced the transcription of BFV LTR promoter [11,23]. The different activities of the NF- $\kappa \mathrm{B}$ pathway in PFV and BFV suggested that it might play different roles in different kinds of FV transcription [17].

BAG3 is a protein that protects IKK- $\gamma$ from proteasome delivery and this delivery results in sustained NF$\kappa \mathrm{B}$ activation and cell survival $[16,25,26]$. It has recently been reported that BAG3 could suppress the transcription of HIV-1 LTR in microglial cell by suppressing the interaction of NF- $\kappa \mathrm{B}$ p65 subunit with the $\kappa B$ DNA motif on HIV LTR [27]. In our study, BAG3 was demonstrated to suppress the transcription of PFV LTR and IP promoter by PFV Tas. While, whether this effect was mediated by NF- $\kappa B$ was open to investigation. It is clear now that $1 \mathrm{NF}-\kappa \mathrm{B}$ binding site was identified on HIV -1 5'LTR and initiation of transactivation by Tat was strongly induced by NF- $\kappa \mathrm{B}$; however NF- $\kappa \mathrm{B}$ binding site was not found in PFV $5^{\prime} \mathrm{LTR}$. Therefore, assumption arose that Tas of PFV and Tat of HIV-1 utilized different transcription regulation approaches. Also, it has been reported that the luciferase activity of HIV-1 LTR reporter vector lacking the NF- $\kappa B$ functional binding site was activated over 100 -fold by coexpression of PFV Tas, while the wild-type HIV-1 LTR was activated only 4 -fold [28]. This suggested that transcription regulation capability of PFV Tas acted in an NF- $\kappa B$-independent way, and this may also explain why 
activating NF- $\kappa \mathrm{B}$ did not pose any positive effect on the transcriptional activity of Tas.

It has been verified that the PMA, one of the activators of the PKC signal pathway, enhanced viral replication in PFV-persistent infected cells [5]. In our study, PMA treatment did exert positive effect on PFV 5'LTR and IP promoter basal and Tas-regulated activity. STS, one of the PKC signal pathway inhibitors showed strong negative effect on LTR basal activity. These results indicated that the PKC signal pathway play a role in PFV replication through regulating the LTR promoter activity. Furthermore, AP-1 protein, which was an important transcription factor involved in the PKC signal pathway, showed a strong positive effect on LTR and IP basal activities but a negative effect on LTR and IP activated promoter activities in our study. Other research studies revealed that AP-1 modulate the IP activity of bovine FV [29]. Also, for HIV-1, the AP-1 binding sites located in the pol gene intragenic regulatory region are important for viral replication [30]. Taken together, AP-1 protein played important roles in retroviruses transcription and replication.

Unexpectedly, during our experiment, the expression level of egrl in Hela cell line transfected with pCI-Tas increased about 3 times than that of the control (unpublished data). Egr1, a nuclear transcriptional regulator involved in the PKC signal pathway, was found to be associated with the HTLV-1Tax-responsive c/s-acting element [31]. Also, Egr1 was reported to be the direct target of the IGE-II gene which was induced by the PKC pathway during $\mathrm{HCV}$ pathogenesis and activated much more immune factors to defend foreign invasion of HCV [32]. These could pave the way for subsequent studies of Egr1 and other cellular regulators, which impacted on specific transcription factor Tas in prototype FV life cycle. But further analysis will be required to evaluate the identities of important transcription factor-binding sites and to identify elements involved in tissue-specific expression of those genes involved in PFV transcription and replication.

\section{Statement of Ethics}

Ethical Approval: This article does not contain any studies with Human Participants or animals.

Informed Consent: This study is exempt from ethical committee approval because this work does not contain any studies with human participants or animals.

\section{Conflict of Interest Statement}

The authors declare that there is no conflict of interest.

\section{Funding Sources}

This work was supported by the grants from the National Natural Science Foundation of China (No. 31670163), the Fundamental Research Funds for the Central Universities (GK201902010, GK202007019), and the Innovative Experiment Projects of Educational Ministry of China for Undergraduate (cx2019098).

\section{Author Contributions}

Y.S. and Z.L. designed the experiments. T.W. conducted the experiments. J.W. analyzed the data and prepared the manuscript. G.Z. assisted with luciferase measurement. W.L. and X.H. gave suggestions on the study.

\section{References}

1 Almodóvar-García K, Kwon M, Samaras SE, Davidson JM, et al. ANKRD1 acts as a transcriptional repressor of MMP13 via the AP-1 site. Mol Cell Biol. 2014;34(8):1500-11.

2 Hu X, Yang W, Liu R, Geng Y, Qiao W, Tan J. N-Myc interactor inhibits prototype foamy virus by sequestering viral Tas protein in the cytoplasm. J Virol. 2014;88(12):7036-44.

3 Bodem J, Kräusslich HG, Rethwilm A. Acetylation of the foamy virus transactivator Tas by PCAF augments promoter-binding affinity and virus transcription. J Gen Virol. 2007; 88(Pt 1):259-63.

4 Kang Y, Blair WS, Cullen BR. Identification and functional characterization of a high-affinity Bel-1 DNA binding site located in the human foamy virus internal promoter. J Virol. 1998;72(1):504-11.
5 Meiering CD, Rubio C, May C, Linial ML. Cell-type-specific regulation of the two foamy virus promoters. J Virol. 2001;75(14):654757.

6 Wagner A, Doerks A, Aboud M, Alonso A, Tokino T, Flügel RM, et al. Induction of cellular genes is mediated by the Bell transactivator in foamy virus-infected human cells. J Virol. 2000;74(10):4441-7.

7 Lee KJ, Lee AH, Sung YC. Multiple positive and negative cis-acting elements that mediate transactivation by bel1 in the long terminal repeat of human foamy virus. J Virol. 1993; 67(4):2317-26.

8 Erlwein O, Rethwilm A. BEL-1 transactivator responsive sequences in the long terminal repeat of human foamy virus. Virology. 1993; 196(1):256-68.
9 Zhu W, Ma H, Miao J, Huang G, Tong M, Zou S. $\beta$-Glucan modulates the lipopolysaccharide-induced innate immune response in rat mammary epithelial cells. Int Immunopharmacol. 2013;15(2):457-65.

10 Sengupta S, Powell E, Kong L, Blackard JT. Effects of HCV on basal and tat-induced HIV LTR activation. PLoS One. 2013;8(6): e64956.

11 Wang J, Tan J, Guo H, Zhang Q, Jia R, Xu X, et al. Bovine foamy virus transactivator BTas interacts with cellular RelB to enhance viral transcription. J Virol. 2010;84(22):1188897.

12 Fedele AO, Carraro V, Xie J, Averous J, Proud CG. Cyclosporin A but not FK506 activates the integrated stress response in human cells. J Biol Chem. 2020;295(44):15134-43. 
13 Gao T, Wang T, Wang Z, Cao J, Dong Y, Chen Y. Melatonin-mediated MT2 attenuates colitis induced by dextran sodium sulfate via $\mathrm{PI3K} / \mathrm{AKT} / \mathrm{Nrf} 2 / \mathrm{SIRT} 1 / \mathrm{ROR} \alpha / \mathrm{NF}-\kappa \mathrm{B}$ signaling pathways. Int Immunopharmacol. 2021;96:107779.

14 Caputto BL, Cardozo Gizzi AM, Gil GA. cFos: an AP-1 transcription factor with an additional cytoplasmic, non-genomic lipid synthesis activation capacity. Biochim Biophys Acta. 2014;1841(9):1241-6.

15 Duverger A, Wolschendorf F, Zhang M, Wagner F, Hatcher B, Jones J, et al. An AP-1 binding site in the enhancer/core element of the HIV-1 promoter controls the ability of HIV-1 to establish latent infection. J Virol. 2013; 87(4):2264-77.

16 Rosati A, Graziano V, De Laurenzi V, Pascale M, Turco MC. BAG3: a multifaceted protein that regulates major cell pathways. Cell Death Dis. 2011;2(4):e141.

17 Kyratsous CA, Silverstein SJ. BAG3, a host cochaperone, facilitates varicella-zoster virus replication. J Virol. 2007;81(14):7491-503.

18 Zhu H, Wu W, Fu Y, Shen W, Miao K, Hong $\mathrm{M}$, et al. Overexpressed BAG3 is a potential therapeutic target in chronic lymphocytic leukemia. Ann Hematol. 2014;93(3):425-35.

19 Kong Q, Hua H, Cui A, Shao T, Song P, Jiang Y. SP600125 induces Src and type I IGF receptor phosphorylation independent of JNK. Int J Mol Sci. 2014;15(9):16246-56.
20 Falco A, Festa M, Basile A, Rosati A, Pascale $\mathrm{M}$, Florenzano F, et al. BAG3 controls angiogenesis through regulation of ERK phosphorylation. Oncogene. 2012;31(50):5153-61.

21 Mergia A, Pratt-Lowe E, Shaw KE, RenshawGegg LW, Luciw PA. cis-acting regulatory regions in the long terminal repeat of simian foamy virus type 1. J Virol. 1992;66(1):251-7.

22 Renne R, Mergia A, Renshaw-Gegg LW, Neumann-Haefelin D, Luciw PA. Regulatory elements in the long terminal repeat (LTR) of simian foamy virus type 3 (SFV-3). Virology. 1993;192(1):365-9.

23 Liu SP, Li XY, Li Z, He LN, Xiao Y, Yan K, et al. Octanoylated ghrelin inhibits the activation of the palmitic acid- induced TLR4/NF$\kappa B$ signaling pathway in THP-1 macrophages. ISRN Endocrinol. 2012;2012:237613.

24 Zhang J, Wang C, Tuo X, Chai K, Xu Y, Qiao $\mathrm{W}$, et al. Prototype foamy virus downregulates RelB expression to facilitate viral replication. FEBS Open Bio. 2020;10(10):2137-48.

25 Ammirante M, Rosati A, Arra C, Basile A, Falco A, Festa M, et al. IKK\{gamma\} protein is a target of BAG3 regulatory activity in human tumor growth. Proc Natl Acad Sci U S A. 2010;107(16):7497-502.

26 Wang HQ, Meng X, Liu BQ, Li C, Gao YY, Niu XF, et al. Involvement of JNK and NF- $\kappa B$ pathways in lipopolysaccharide (LPS)-induced BAG3 expression in human monocytic cells. Exp Cell Res. 2012;318(1):16-24.
27 Bruno AP, De Simone FI, Iorio V, De Marco M, Khalili K, Sariyer IK, et al. HIV-1 Tat protein induces glial cell autophagy through enhancement of BAG3 protein levels. Cell $\mathrm{Cy}$ cle. 2014;13(23):3640-4.

28 Keller A, Garrett ED, Cullen BR. The Bel-1 protein of human foamy virus activates human immunodeficiency virus type 1 gene expression via a novel DNA target site. J Virol. 1992;66(6):3946-9.

29 Wu Y, Tan J, Su Y, Qiao W, Geng Y, Chen Q. Transcription factor AP1 modulates the internal promoter activity of bovine foamy virus. Virus Res. 2010;147(1):139-44.

30 Colin L, Vandenhoudt N, de Walque S, Van Driessche B, Bergamaschi A, Martinelli V, et al. The AP-1 binding sites located in the pol gene intragenic regulatory region of HIV-1 are important for viral replication. PLoS One. 2011;6(4):e19084.

31 Huang Q, Niu Z, Han J, Liu X, Lv Z, Li H, et al. HTLV-1 Tax upregulates early growth response protein 1 through nuclear factor- $\kappa \mathrm{B}$ signaling. Oncotarget. 2017;8(31):51123-33.

32 Lee S, Park U, Lee YI. Hepatitis C virus core protein transactivates insulin-like growth factor II gene transcription through acting concurrently on Egr1 and Sp1 sites. Virology. 2001;283(2):167-77.

33 Wang XF, Liu XJ, Zhou QM, Du J, Zhang TL, $\mathrm{Lu}$ YY, et al. Ginsenoside rb1 reduces isoproterenol-induced cardiomyocytes apoptosis in vitro and in vivo. Evid Based Complement $\mathrm{Al}-$ ternat Med. 2013;2013:454389. 